\title{
Three Nonlinear NDE Techniques On Three Diverse Objects
}

\author{
Kristian C.E Haller, and Claes M. Hedberg \\ Blekinge Institute of Technology, 37179 Karlskrona, Sweden
}

\begin{abstract}
Non-Destructive Evaluation has been carried out on three different test objects, with three different methods based on exhibits of slow dynamics and nonlinear effects. The three diverse objects were cast iron, ceramic semi-conductors on circuit boards, and rubber. The three approaches were Higher Harmonics detection $(\mathrm{HH})$, Nonlinear Wave Modulation Spectroscopy (NWMS), and Slow Dynamics (SD). For all of the objects the three approaches were tried. The results showed that for each of the objects, a different method worked the best. The cast iron worked best with nonlinear wave modulation, the ceramic semi-conductors worked well with the higher harmonics detection, while the rubber showed best results with slow dynamics.
\end{abstract}

Keywords: Nondestructive testing, nonlinear acoustics, higher harmonics, nonlinear wave modulation spectroscopy, slow dynamics.

PACS: 43.25 .Dc

\section{INTRODUCTION}

Sensitive nonlinear methods are in strong progressive development. High sensitivity gives the opportunity to find defects and characterize the material characterization in a very careful manner. The Higher Harmonic ( $\mathrm{HH})$ [1] method has been used in evaluation for some time. Nonlinear Wave Modulation Spectroscopy (NWMS) [2] and Slow Dynamics (SD) [3], [4] have been introduced in later years. In this work, all three of these methods have been applied to diverse objects to evaluate the objects damage and to determine what method is best to use. Three diverse objects were examined and they are from different industrial areas.

One object is a cast iron steel part of a large cutting tool. First an intact undamaged part was tested to find out if the response was nonlinear from preexisting cracks and defects. A damage was introduced at the top of the cutting edge and then the result of the new measurement is compared to the one for the undamaged.

Ceramic semiconductors used for capacitive loads are very common in electrical components and are manufactured as standard components to be machine mounted on printed circuits. These semiconductors sometimes break partially and can give intermittent errors. The ceramic semiconductors were bent to introduce cracks and defects but since they are brittle they tend to break completely (instead of partly cracking). A solution was chosen where the piezo ceramics are bonded to the printed circuit. Damage is introduced while the ceramic semiconductors are still held in place by its soldering spots.

CP838, Innovations in Nonlinear Acoustics: $17^{\text {th }}$ International Symposium on Nonlinear Acoustics, edited by A. A. Atchley, V. W. Sparrow, and R. M. Keolian

(C) 2006 American Institute of Physics 0-7354-0330-9/06/\$23.00 
Rubber for cable transmission sealing through walls is manufactured in blocks and then cut to desired size and shape. Before the cut operation one need to determine the quality and homogeneity of the block. Two different batches from the production were tested, one of good quality and another less good.

\section{EXPERIMENTAL METHODOLOGY AND RESULTS}

These experiments are all conducted using commercially available piezoceramic transducers (PZT) glued with epoxy. One PZT is acting as an actuator to generate the acoustic wave in the specimen and the other is acting as a pickup of the acoustical wave.

For the higher harmonics measurement a single sinusoid acoustic wave is sent to the specimen by the signal generator through one PZT. The signal is distorted by cracks and other defects on its way to the PZT acting as a sensor. In the recorded frequency spectrum higher harmonics are detected to indicating the amount of cracks or defects.

For the Nonlinear Wave Modulation Spectroscopy (NWMS), a single frequency signal is repeated as for $(\mathrm{HH})$, but now with an addition of some of the specimen's own resonant frequencies, lower in frequency than the single high frequency signal. This is accomplished by hitting the object with a test hammer. In the recorded frequency spectrum sidebands appear beside the single frequency if defects and cracks are present.

The Slow Dynamics time signal method (SD) is the monitoring of the resonance frequency after a material is conditioned by a transient force. If the material is damaged, the resonance frequency is found to drop instantaneously and then the resonance frequency is slowly recovering - logarithmically with time - to its initial value.

All methods were applied on all objects for evaluation. For each object the best suited method is presented, with results from the other methods briefly commented.

For the cast iron cutting tool the NWMS method was best, see Figure 1. The material itself shows nonlinear response in the undamaged state, indicated by all three methods. This was an expected result because casting iron is a complicated process and inhomogeneities and inclusions can be problematic for this process. Calculating the sideband energy and comparing the undamaged and damaged states showed a high difference in values. The HH showed differences in number of harmonics and in their amplitude, but was not as clear as NWMS. The difference in SD indicated defect, but was not large. 


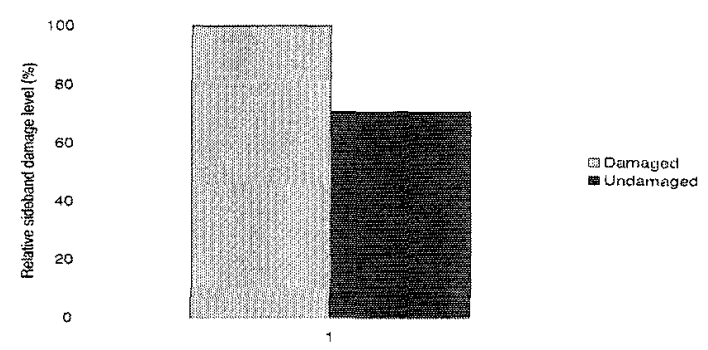

FIGURE 1. Nonlinear Wave Modulation Spectroscopy results for cast iron shown as sideband energy for undamaged in the right column, and damaged in the left column.

The ceramic-semiconductors mounted to the printed circuit board showed the $\mathrm{HH}$ worked best. Higher harmonics was present even when the semiconductors not was damaged. This indicates that either the board itself gives nonlinear response or some component mounted was damaged, but testing a larger number of boards indicates the nonlinear response arises from the board. When damaging the semiconductors the number of multiple harmonics increases also the amplitude of the harmonics increases, Figure 2. The results from NWMS was indicating defects but not as strong as the $\mathrm{HH}$. Also SD was indicating defects but not as strong as $\mathrm{HH}$.

Harmonic distortion

Harnonic distorion
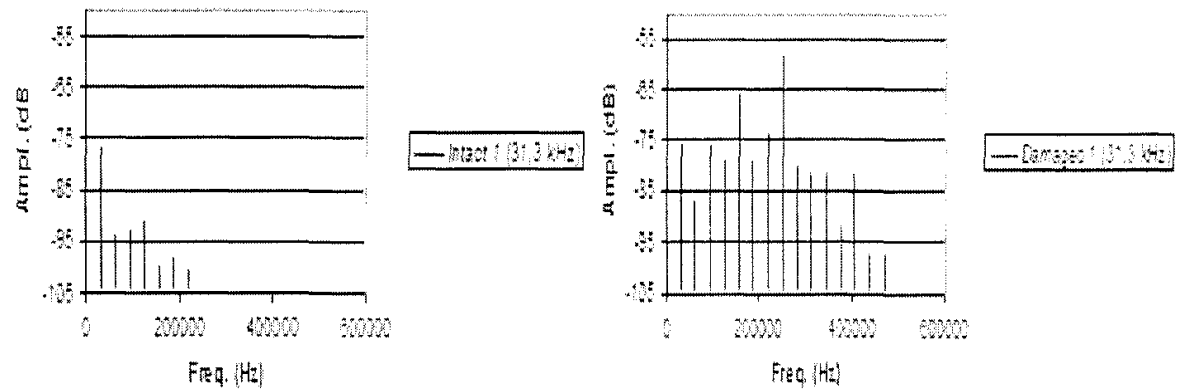

FIGURE 2. Higher Harmonics results of an undamaged circuit board ceramics on the left, and a damaged one on the right.

For the rubber bricks the SD method was the only one to indicate damage and quality differences, Figure 3. NWMS gave no measurable differences in sideband energy. No clear differences in $\mathrm{HH}$ could give results to conclude material quality differences. 

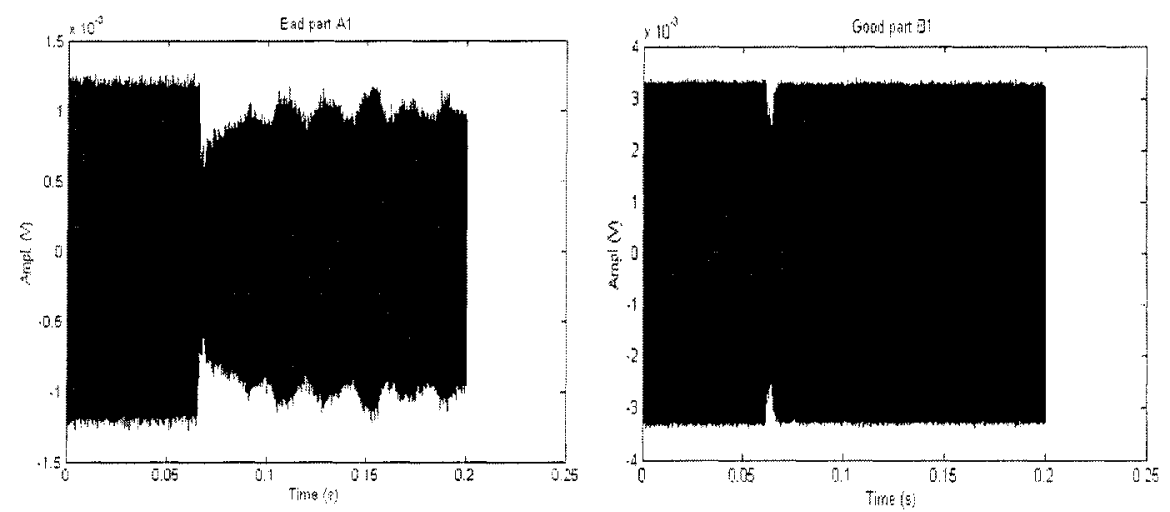

FIGURE 3. Slow Dynamics time signal results of good quality rubber on the left, and bad quality rubber on the right.

\section{DISCUSSION}

The method of Higher Harmonics (HH) was succesfully applied to circuit boards for detection of damage of ceramic semi-conductors. For circuit boards the method with no impacts is convenient when one would like to be careful with the components. The Nonlinear Wave Modulation Spectroscopy (NWMS) method worked very good with the cast iron. Rubber was an unexperienced area for the authors before conducting these experiment. Remarkably only Slow Dynamics (SD) could detect differences. It is known $\mathrm{SD}$ is a very sensitive method, but the other methods gave no indication to separate between the different samples. This is the first time we have encountered the case where SD yields high response, while the fast dynamics methods (NWMS and $\mathrm{HH}$ ) would not indicate any change with damage.

\section{ACKNOWLEDGEMENTS}

This work is financed by the grant "Nonlinear nondestructive evaluation of material conditions - resonance and pulse techniques" from Vetenskapsrådet (Sweden). We would like to thank the TANGO-Verkstad for project support. The European Science Foundation programme NATEMIS (Nonlinear Acoustic Techniques for Micro-Scale Damage Diagnostics) is also acknowledged.

\section{REFERENCES}

1. Buck,O., Morris,W.L. and Richardson,J.M., Appl.Phys.Lett. 33. 371-372 (1978).

2. Van Den Abeele, K.E.-A., Johnson, P.A. and Sutin, A., Res.Nondestr.Eval., 12, 17 - 30 (2001).

3. R.A.Guyer and P.A.Johnson, Physics Today, April 1999, 30-36.

4. TenCate, J.A., and Shankland, T.J., Geophys.Res.Lett. 23(21), 3019-3022 (1996). 